\title{
Rurality and dietary patterns: associations in a UK cohort study of 10-year-old children
}

\author{
Tim T Morris* and Kate Northstone \\ School of Social and Community Medicine, University of Bristol, Oakfield House, Oakfield Grove, \\ Bristol BS8 2BN, UK
}

Submitted 24 February 2014: Final revision received 16 May 2014: Accepted 23 July 2014: First published online 5 September 2014

\begin{abstract}
Objective: Despite differences in obesity and ill health between urban and rural areas in the UK being well documented, very little is known about differences in dietary patterns across these areas. The present study aimed to examine whether urban/rural status is associated with dietary patterns in a population-based UK cohort study of children.

Design: Dietary patterns were obtained using principal components analysis and cluster analysis of $3 \mathrm{~d}$ diet records collected from children at 10 years of age. Rurality was obtained from the 2001 UK Census urban/rural indicator at the time of dietary assessment. General linear models were used to examine the relationship between rurality and dietary pattern scores from principal components analysis; multinomial logistic regression was used to assess the association between rurality and dietary clusters.

Setting: The Avon Longitudinal Study of Parents and Children (ALSPAC), South West England.

Subjects: Children ( $n$ 5677) aged 10 years (2817 boys and 2860 girls).

Results: After adjustment, increases in rurality were associated with increased scores on the 'health awareness' dietary pattern $(\beta=0.35 ; 95 \%$ CI $0.14,0.56$; $P<0.001$ for the most rural compared with the most urban group) and lower scores on the 'packed lunch/snack' dietary pattern $(\beta=-0.39 ; 95 \% \mathrm{CI}-0.59$, $-0.19 ; P<0.001$ for the most rural compared with the most urban group). The odds ratio for participants being in the 'healthy' compared with the 'processed' dietary cluster for the most rural areas was 1.61 (95\% CI 1.05, 2.49; $P=0.02$ ) compared with those in the most urban areas.

Conclusions: There is evidence to suggest that differences exist in dietary patterns between rural and urban areas. Similar results were found using two different methods of dietary pattern analysis, showing that children residing in rural households were more likely to consume healthier diets than those in urban households.
\end{abstract}

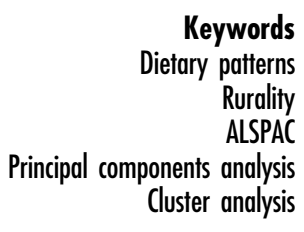

Keywords

Rurality

ALSPAC

Cluster analysis
Obesity continues to rise in the $\mathrm{UK}^{(1)}$, posing a significant threat to the health of the nation. While there are many facets to the obesity epidemic ${ }^{(2)}$, multiple interventions and policy changes have failed to halt the continued rise over the past few years. Rates of obesity ${ }^{(3)}$ (and poor health in general $^{(4)}$ ) in the UK vary considerably between rural and urban areas, whereby people living in rural areas are consistently healthier than their urban-dwelling counterparts. A greater understanding of this marked geographical difference may better inform policy interventions that are more effective at tackling the underlying causes and reducing the prevalence of obesity in the UK.

Diet plays a vital role in the development of obesity and numerous other health problems ${ }^{(5)}$. Poor diet quality is cited as a major driver behind the obesity epidemic and is a highly modifiable risk factor for obesity and ill health $^{(6)}$. This is particularly important for the development of children given that dietary habits formed in childhood and adolescence have been shown to track into adulthood $^{(7,8)}$. The role of diet in obesity development therefore makes it a sensible policy intervention target for improving the health of the nation in an attempt to halt and potentially reverse the obesity epidemic. For effective policy intervention though, it may be necessary to understand not only to whom health interventions can be most effectively targeted, but also geographically where they would be most effective; for instance, areas where dietary patterns tend to be of poorer quality. It is therefore 
important that geographical variations in diet - as an underlying driver of obesity and ill health - are well understood.

A number of studies have identified the influence that space and place have on dietary intake, both internationally ${ }^{(9-11)}$ and in the $\mathrm{UK}^{(12-14)}$. However, research has focused largely on one of two factors: (i) the effects of neighbourhood-level deprivation ${ }^{(15-18)}$; and (ii) the role that distance or access to food outlets plays in diet ${ }^{(19-24)}$. Results from UK studies have been mixed ${ }^{(25)}$, indicating that geographical differences observed in neighbourhood diet quality may not be due to deprivation or affluence once individual-level variables such as income, education and class are accounted for and may not be due to accessibility or price either. This is understandable, as an increased choice of food outlets in the immediate local vicinity selling cheap healthy foods does not necessarily translate to a change in dietary habits. Habits tend to be formed over long periods of time from early-life experiences $^{(7,8)}$.

There has been very limited research dedicated to examining differences in dietary patterns between different rural/urban profiles of the UK. Given the documented differences in obesity and ill health between rural and urban residents in the UK, whereby residents of urban areas suffer poorer health than those from rural areas ${ }^{(3,4)}$, one may expect that diet quality varies geographically in a similar manner between urban and rural areas. Recent research from both the $\mathrm{USA}^{(26,27)}$ and Canada ${ }^{(28)}$ shows that in a North American context increasing rurality is associated with poorer nutrient profiles consisting of higher consumption of high-fat and high-sugar foods. To our knowledge, however, little research has been conducted examining dietary intake and rurality in the UK, where urban/rural profiles clearly differ from Canada and the USA in terms of population demographics, income disparity and service provision. UK-based evidence shows a higher prevalence of more 'traditional' dietary patterns (based on core foods such as bread, potatoes, vegetables) in rural areas ${ }^{(29)}$, and that adults in rural households and students in rural schools have higher fruit and vegetable intakes than their urban counterparts ${ }^{(30,31)}$ despite poorer access to food in rural areas ${ }^{(17)}$. Research from other European countries also suggests a greater prevalence of higher-quality diets in rural households ${ }^{(32)}$.

The use of dietary patterns that reflect the total diet, as an alternative or complementary approach to studying the associations between diet and health, has increased considerably over recent years. These methods recognise that foods and drinks are consumed in combination and enable the study of the whole diet, rather than individual foods or nutrients. Two common methods for deriving dietary patterns are principal components analysis (PCA) and cluster analysis. PCA uses the correlations that exist between different food groups to identify linear combinations of foods that are frequently consumed together and gives each individual a score for every component.
Cluster analysis, however, combines individuals into nonoverlapping groups based on similarity of dietary intakes. To our knowledge, only two studies have been performed specifically examining UK differences in dietary patterns with a focus on rurality ${ }^{(29,30)}$, but neither of these focused on children. The dietary patterns used in the present paper had already been obtained using both PCA and cluster analysis from dietary data collected from the study cohort using diet diaries at 10 years of age ${ }^{(33,34)}$.

Given the lack of UK-specific research on differences in dietary patterns between urban and rural areas, the aim of the present study was to examine whether the urban/rural indicator is associated with dietary patterns in a populationbased UK cohort study of children.

\section{Methods}

Subjects were children from the Avon Longitudinal Study of Parents and Children (ALSPAC). Pregnant women were eligible to join the study if they had an expected date of delivery between April 1991 and December 1992 and were resident in the (former) Avon Health Authority area in South West England ${ }^{(35)}$. A total sample of 14701 children was used, including 13988 children from the originally recruited sample who were still alive at 1 year of age and an additional 713 children who were recruited at later ages. The ALSPAC cohort is largely representative of the UK when compared with 1991 Census data, with slight under-representation in ethnic minorities, single-parent families and those living in rented accommodation. Ethical approval for the study was obtained from the ALSPAC Ethics and Law Committee and the Local Research Ethics Committees. Please note that the study website contains details of all the data that are available through a fully searchable data dictionary ${ }^{(36)}$.

The dietary assessment at 10 years of age has been described in detail elsewhere ${ }^{(37)}$. Briefly, children were invited to attend a hands-on dietary assessment in a clinic setting. Prior to the visit children were asked to record all foods and drinks that had been consumed over three full days (two weekdays and one weekend day). At the clinic children were interviewed by a nutrition fieldworker to allow the inclusion of any food that had been missed out and obtain a greater description of any portion sizes and cooking methods. Twenty-four-hour recalls were conducted by the fieldworkers in the event that a child had attended a clinic without a complete diet diary. Coding was completed by the fieldworker using the DIDO (Diet In, Data Out) software program developed by the MRC Human Nutrition Research Unit (Cambridge, UK).

\section{Exposure}

The 2001 Census urban/rural indicator (URI) was applied to ALSPAC geographic data; month and year of the clinic visit date were used to extract the relevant household 
residence from the ALSPAC address table by census Output Area (OA). 2001 OA contain a minimum of forty households and 100 people and are built from clusters of neighbouring postcode units; they avoid urban/rural mixes where possible and are designed to be socially homogeneous while maintaining similar population counts ${ }^{(38)}$. The URI was designed to permit research analysis 'according to different types of rural and urban area ${ }^{(39)}$ and as such separates English, Welsh and Scottish addresses by OA into four categories based upon population density: (i) 'urban $>10000$ ', (ii) 'town and fringe', (iii) 'village' and (iv) 'hamlet and isolated dwelling', before splitting each of these categories into whether surrounding areas are either 'sparse[ly]' or 'less sparse[ly]' populated, to give a total of eight categories $^{(39)}$. Few children fell into the 'sparse' categories ( $n$ 30), so URI values were recoded to include both sparse and less sparse in each rurality category to give a total of four categories for analysis.

\section{Outcomes}

Outcomes of interest were dietary patterns obtained through both PCA and cluster analysis. The patterns were derived from $3 \mathrm{~d}$ diet record data using gram weight $(\mathrm{g} / \mathrm{d})$ as input variables and have been described in detail elsewhere ${ }^{(34)}$. Three dietary patterns were derived from PCA; (i) one contrasting more healthy (high-fibre bread, vegetables, water) with less healthy/processed foods (processed foods, chips, soft drinks; labelled 'health awareness'); (ii) one with high loadings on traditional British foods (meat, potatoes and vegetables; labelled 'traditional'); and (iii) the last with high loadings on foods that are often found in a child's packed lunch such as white bread, sandwich fillings and snacks such as crisps and chocolate (labelled 'packed lunch/snack').

Four clusters were derived from the same data using $k$-means cluster analysis ${ }^{(33)}$; these were labelled 'processed', 'healthy', 'traditional' and 'packed lunch' according to the average intake of foods consumed by the children falling into each cluster.

\section{Potential confounders}

Potential confounders were obtained from self-completion questionnaires and included highest maternal education at pregnancy ('low' (less than O-level, where O-level represents qualifications obtained at 16 years of age), 'middle' (O-level) and 'high' (higher than O-level, i.e. education beyond 16 years of age)); maternal age at delivery $(<25$ years, 25-29 years, 30-34 years and 35+ years); highest parental social class based on occupation ${ }^{(40)}$ as reported by the mother in pregnancy (with social class I as 'high' and social classes IV and V as 'low' (social classes IV and $\mathrm{V}$ were grouped together due to low cell counts in the most rural areas)); gender; and home ownership status reported at 10 years (categorised as owned/mortgaged, rented from council or housing association, and rented privately/other).

\section{Statistical methods}

Differences in mean PCA scores according to URI were examined using ANOVA while a $\chi^{2}$ test was used to establish any difference between dietary cluster membership and URI. Multivariable linear regression analysis was used to estimate the effects of URI on each of the scores from PCA separately; first unadjusted and then adjusted for all potential confounders. Finally, multinomial logistic regression was used to determine relationships between level of rurality and dietary cluster membership, again employing both unadjusted models and those adjusted for confounders to obtain odds ratios and $95 \%$ confidence intervals. Throughout 'urban' was used as the reference category. All statistical analyses were performed using the statistical software package STATA version 12 .

\section{Results}

Of the 11868 children invited to attend the clinic visit at 10 years of age, 7557 (64\%) attended, of whom 7473 provided dietary data ( 3702 boys and 3771 girls). Of those with dietary data, a further 1796 (24\%) children were excluded due to missing data on the exposure variable ( $n$ 61) or from one of the confounder variables ( $n$ 1735), leaving a total of 5677 cases for analysis. Missing data from the exposure variable was due to minor historical database migration errors resulting in no valid address and therefore no URI value attached. Twenty-four-hour recalls were conducted for 610 children $(10.7 \%)$ with no difference observed between children from different areas in terms of rurality $(P=0.954)$.

Table 1 displays the differences in baseline characteristics of the cohort between those from different areas in terms of rurality. Differences were observed between rurality categories for maternal education, maternal age, social class and home ownership (all $P<0 \cdot 004$ ). These differences indicate that children from rural areas were more likely to come from higher class and better educated families who owned/mortgaged their homes, and have older mothers than their more urban counterparts.

It can be seen in Table 2 that increasing rurality was associated with increases in mean 'health awareness' and 'traditional' dietary pattern scores and a decrease in mean 'packed lunch/snack' score. Table 3 shows that children living in increasingly urban areas were more likely to belong to the 'processed' and 'packed lunch' clusters. Conversely, children living in increasingly rural areas were more likely to belong to the 'healthy' and 'traditional' clusters.

Table 4 shows that unadjusted parameter estimates increased for the 'health awareness' dietary pattern with increasing rurality. Although the estimates were attenuated, the trend was still evident after adjustment $(P<0 \cdot 001)$. Conversely, the 'packed lunch/snack' dietary pattern showed a negative trend both before and after adjustment with increasing rurality $(P<0 \cdot 001)$. There appeared to be 
Table 1 Differences in baseline characteristics for ALSPAC children with complete data by URI categories

\begin{tabular}{|c|c|c|c|c|c|c|c|c|}
\hline & \multicolumn{2}{|c|}{ Urban } & \multicolumn{2}{|c|}{ Town } & \multicolumn{2}{|c|}{ Village } & \multicolumn{2}{|c|}{ Hamlet } \\
\hline & $n$ & $\%$ & $n$ & $\%$ & $n$ & $\%$ & $n$ & $\%$ \\
\hline Total & 4623 & 81.43 & 390 & $6 \cdot 87$ & 488 & 8.60 & 176 & $3 \cdot 10$ \\
\hline \multicolumn{9}{|l|}{ Gender } \\
\hline Boy $(n 2817)$ & 2281 & $49 \cdot 34$ & 198 & $50 \cdot 77$ & 246 & $50 \cdot 41$ & 92 & $52 \cdot 27$ \\
\hline $\begin{array}{l}\text { Girl }(n 2860) \\
x^{2}=0.968(P=0.809)\end{array}$ & 2342 & $50 \cdot 66$ & 192 & $49 \cdot 23$ & 242 & 49.59 & 84 & $47 \cdot 73$ \\
\hline \multicolumn{9}{|l|}{ Maternal education } \\
\hline Low $(n 1105)$ & 987 & 21.35 & 43 & 11.03 & 51 & $10 \cdot 45$ & 24 & $13 \cdot 64$ \\
\hline Middle ( $n$ 2022) & 1698 & 36.73 & 131 & 33.59 & 150 & $30 \cdot 74$ & 43 & 24.43 \\
\hline $\begin{array}{l}\text { High }(n 2550) \\
x^{2}=108.722(P<0.001)\end{array}$ & 1938 & 41.92 & 216 & $55 \cdot 38$ & 287 & $58 \cdot 81$ & 109 & 61.93 \\
\hline \multicolumn{9}{|l|}{ Maternal age at delivery (years) } \\
\hline$<25(n 716)$ & 628 & 13.58 & 32 & $8 \cdot 21$ & 38 & 7.79 & 18 & $10 \cdot 23$ \\
\hline $25-29(n 2306)$ & 1893 & 40.95 & 162 & 41.54 & 202 & 41.39 & 49 & 27.84 \\
\hline 30-34 (n 1951) & 1550 & 33.53 & 148 & 37.95 & 183 & 37.50 & 70 & 39.77 \\
\hline $\begin{array}{l}35+(n 704) \\
x^{2}=46.556(P<0.001)\end{array}$ & 552 & 11.94 & 48 & $12 \cdot 31$ & 65 & 13.32 & 39 & $22 \cdot 16$ \\
\hline \multicolumn{9}{|l|}{ Highest parental social class } \\
\hline I ( $n 932)$ & 689 & 14.90 & 67 & $17 \cdot 18$ & 120 & 24.59 & 56 & 31.82 \\
\hline II $(n$ 2583) & 2018 & 43.65 & 213 & 54.62 & 269 & $55 \cdot 12$ & 83 & $47 \cdot 16$ \\
\hline III non-manual ( $n$ 1398) & 1238 & $26 \cdot 78$ & 73 & $18 \cdot 72$ & 67 & $13 \cdot 73$ & 20 & $11 \cdot 36$ \\
\hline III manual ( $n$ 551) & 489 & 10.58 & 28 & $7 \cdot 18$ & 25 & $5 \cdot 12$ & 9 & $5 \cdot 11$ \\
\hline IV \& V $(n 213)$ & 189 & 4.09 & 9 & 2.31 & 7 & 1.43 & 8 & 4.55 \\
\hline$x^{2}=154.247(P<0.001)$ & & & & & & & & \\
\hline \multicolumn{9}{|l|}{ Home ownership status } \\
\hline Owned/mortgaged ( $n$ 5122) & 4145 & 89.66 & 358 & 91.79 & 458 & 93.85 & 161 & 91.48 \\
\hline Rented privately/other $(n 124)$ & 96 & 2.08 & 11 & $2 \cdot 82$ & 13 & 2.66 & 4 & 2.27 \\
\hline $\begin{array}{l}\text { Rented from council or housing association ( } n \text { 431) } \\
x^{2}=19.013(P=0.004)\end{array}$ & 382 & $8 \cdot 26$ & 21 & $5 \cdot 38$ & 17 & 3.48 & 11 & $6 \cdot 25$ \\
\hline
\end{tabular}

ALSPAC, Avon Longitudinal Study of Parents and Children; URI, urban/rural indicator.

Table 2 Dietary pattern scores (mean and standard deviation) obtained from PCA at 10 years of age among ALSPAC children by URI category

\begin{tabular}{|c|c|c|c|c|c|c|}
\hline & \multicolumn{2}{|c|}{ 'Health awareness' dietary pattern } & \multicolumn{2}{|c|}{ 'Traditional' dietary pattern } & \multicolumn{2}{|c|}{ 'Packed lunch/snack' dietary patterr } \\
\hline & Mean & SD & Mean & SD & Mean & SD \\
\hline Urban & -0.08 & 1.5 & 0.02 & 1.46 & 0.06 & 1.34 \\
\hline Town & 0.29 & 1.48 & -0.03 & 1.41 & -0.09 & 1.25 \\
\hline Village & 0.42 & 1.55 & -0.02 & 1.47 & -0.23 & 1.31 \\
\hline Hamlet & 0.62 & 1.43 & 0.23 & 1.41 & -0.42 & 1.37 \\
\hline$P$ value & $<0.001$ & & 0.173 & & $<0.001$ & \\
\hline
\end{tabular}

PCA, principal components analysis; ALSPAC, Avon Longitudinal Study of Parents and Children; URI, urban/rural indicator.

little difference in 'traditional' dietary pattern scores for children living in different areas.

Table 5 presents the results from the multinomial logistic regression presenting the odds ratios for being in the 'healthy', 'traditional' or 'packed lunch' cluster compared with the 'processed' cluster. The odds of being in the 'healthy' cluster compared with the 'processed' cluster increased with increasing rurality, with children living in hamlets/isolated dwellings being more than twice as likely to be in the 'healthy' cluster (OR $=2 \cdot 24 ; 95 \%$ CI 1.48, 3.37). This association was attenuated after adjustment but a trend remained with increasing rurality (hamlet $v$. urban: adjusted $\mathrm{OR}=1 \cdot 61 ; 95 \%$ CI 1.05, 2.49). Results showed a small association between increased rurality and belonging to the 'traditional cluster' but the strength of these associations was weak $(P=0 \cdot 183)$. There was no evidence to suggest that URI was associated in any way with being in the 'packed lunch' cluster compared with the 'processed' cluster.

\section{Discussion}

The present study explored urban/rural differences in dietary patterns obtained through both PCA and cluster analysis. The results indicate that rurality is associated with dietary patterns assessed using two distinct methods, even after adjustment for potential confounding factors. We have shown that rurality is associated with diet whereby children living in the most rural areas are more likely to consume what may be considered to be a healthier diet. 
Table 3 Associations between clusters of dietary pattern at 10 years of age among ALSPAC children and URI $\left(X^{2}=76 \cdot 10, P<0 \cdot 001\right)$

\begin{tabular}{|c|c|c|c|c|c|c|c|c|}
\hline & \multicolumn{2}{|c|}{ 'Processed' cluster } & \multicolumn{2}{|c|}{ 'Healthy' cluster } & \multicolumn{2}{|c|}{ 'Traditional' cluster } & \multicolumn{2}{|c|}{ 'Packed lunch' cluster } \\
\hline & $n$ & $\%$ & $n$ & $\%$ & $n$ & $\%$ & $n$ & $\%$ \\
\hline Urban & 1283 & $29 \cdot 61$ & 1196 & $27 \cdot 60$ & 937 & 21.62 & 917 & $21 \cdot 16$ \\
\hline Town & 93 & $25 \cdot 14$ & 132 & 35.68 & 74 & 20.00 & 71 & 19.19 \\
\hline Village & 110 & $23 \cdot 76$ & 175 & 37.80 & 95 & 20.52 & 83 & 17.93 \\
\hline Hamlet & 35 & 20.47 & 73 & 42.69 & 43 & 25.15 & 20 & 11.70 \\
\hline Total & 1521 & 28.50 & 1576 & 29.53 & 1149 & 21.53 & 1091 & 20.44 \\
\hline
\end{tabular}

ALSPAC, Avon Longitudinal Study of Parents and Children; URI, urban/rural indicator.

Table 4 Unadjusted and adjusted ${ }^{*}$ parameter estimates (and $95 \%$ confidence intervals) for the associations between URI and dietary pattern scores obtained from PCA at 10 years of age among ALSPAC children

\begin{tabular}{|c|c|c|c|c|c|c|}
\hline & \multicolumn{2}{|c|}{ 'Health awareness' dietary pattern } & \multicolumn{2}{|c|}{ 'Traditional' dietary pattern } & \multicolumn{2}{|c|}{ 'Packed lunch/snack' dietary patterr } \\
\hline & $\beta$ & $95 \% \mathrm{Cl}$ & $\beta$ & $95 \% \mathrm{Cl}$ & $\beta$ & $95 \% \mathrm{Cl}$ \\
\hline \multicolumn{7}{|c|}{ Unadjusted ( $n$ 5677) } \\
\hline Urban & 0.00 & & 0.00 & & 0.00 & \\
\hline Town & 0.30 & $0.14,0.45$ & -0.04 & $-0.19,0.11$ & -0.15 & $-0.29,-0.1$ \\
\hline Village & 0.43 & $0.29,0.57$ & -0.03 & $-0.17,0.01$ & -0.28 & $-0.41,-0.16$ \\
\hline Hamlet & 0.62 & $0.39,0.85$ & 0.22 & $0.00,0.44$ & -0.49 & $-0.69,-0.28$ \\
\hline$P$ value & $<0.001$ & & 0.21 & & $<0.001$ & \\
\hline \multicolumn{7}{|c|}{ Adjusted $^{*}(n$ 5677) } \\
\hline Urban & 0.00 & & 0.00 & & 0.00 & \\
\hline Town & 0.14 & $0.00,0.29$ & -0.02 & $-0.18,0.13$ & -0.11 & $-0.25,0.03$ \\
\hline Village & 0.21 & $0.08,0.34$ & 0.00 & $-0.14,0.14$ & -0.22 & $-0.35,-0.10$ \\
\hline Hamlet & 0.35 & $0.14,0.56$ & 0.27 & $0.05,0.49$ & -0.39 & $-0.59,-0.19$ \\
\hline$P$ value & $<0.001$ & & 0.12 & & $<0.001$ & \\
\hline
\end{tabular}

URI, urban/rural indicator; PCA, principal components analysis; ALSPAC, Avon Longitudinal Study of Parents and Children.

${ }^{*}$ Adjusted for gender, maternal education, maternal age at delivery, highest parental social class and home ownership status.

Table 5 Unadjusted and adjusted* odds ratios (and $95 \%$ confidence intervals) for the associations between URI and clusters of dietary pattern at 10 years of age among ALSPAC children, using the 'processed' cluster as base outcome

\begin{tabular}{|c|c|c|c|c|c|c|}
\hline & \multicolumn{2}{|c|}{ 'Healthy' cluster } & \multicolumn{2}{|c|}{ 'Traditional' cluster } & \multicolumn{2}{|c|}{ 'Packed lunch' cluster } \\
\hline & OR & $95 \% \mathrm{Cl}$ & OR & $95 \% \mathrm{Cl}$ & OR & $95 \% \mathrm{Cl}$ \\
\hline \multicolumn{7}{|c|}{ Unadjusted ( $n$ 5337) } \\
\hline Urban & 1.00 & - & 1.00 & - & 1.00 & - \\
\hline Town & 1.52 & $1 \cdot 15,2 \cdot 01$ & 1.09 & $0.79,1.50$ & 1.07 & $0.78,1.47$ \\
\hline Village & $1 \cdot 71$ & $1 \cdot 33,2 \cdot 19$ & 1.18 & $0.89,1.58$ & 1.06 & $0.78,1.42$ \\
\hline Hamlet & $2 \cdot 24$ & $1.48,3.37$ & 1.68 & $1.07,2.65$ & 0.80 & $0.46,1.39$ \\
\hline$P$ value & $<0.001$ & & 0.10 & & 0.82 & \\
\hline \multicolumn{7}{|c|}{ Adjusted* (n 5337) $^{*}$} \\
\hline Urban & 1.00 & - & 1.00 & - & 1.00 & - \\
\hline Town & 1.27 & $0.95,1.69$ & 1.04 & $0.76,1.43$ & 1.04 & $0.76,1.43$ \\
\hline Village & $1 \cdot 30$ & $1.00,1.68$ & $1 \cdot 12$ & $0.84,1.50$ & 1.01 & $0.75,1.37$ \\
\hline Hamlet & 1.61 & $1.05,2.49$ & 1.63 & $1.03,2.58$ & 0.79 & $0.45,1.37$ \\
\hline$P$ value & 0.02 & & 0.18 & & 0.85 & \\
\hline
\end{tabular}

URI, urban/rural indicator; ALSPAC, Avon Longitudinal Study of Parents and Children.

${ }^{*}$ Adjusted for gender, maternal education, maternal age at delivery, highest parental social class and home ownership status. Note: 340 children with dietary data were unassigned to food clusters ( 290 from 'urban', 20 from 'town', 25 from 'village', 5 from 'hamlet').

Results using the PCA scores showed that children living in the most rural areas were more likely to consume a healthy diet than those in urban areas, with increased health awareness scores and lower packed lunch/snack scores. There was evidence of a dose-response relationship between both health awareness and packed lunch/ snack scores with increasing rurality.
Cluster analysis identified similar patterns to those from the PCA and the associations with rurality were mirrored. Children residing in rural areas were more likely to belong to the healthy cluster than those in urban areas. As with the PCA results, the likelihood of being in the healthy category increased with increasing rurality. Cluster analysis results from the traditional cluster were weak after 
adjustment for socio-economic characteristics but there was evidence to suggest that children in the most rural areas were more likely to fall into that cluster than children in more urban areas.

More informed knowledge on differences in dietary intake is of critical importance if we are to accurately and reliably identify the underlying mechanisms that may drive such differences between areas of differing rural profiles and help to further explain those that have been seen between diet and deprivation or access to food. Our findings show the opposite trends to those shown by Downs et al. in Canada ${ }^{(28)}$ but they are consistent with UK findings investigating aspects of diet quality and rurality ${ }^{(30,31)}$. These latter studies reported a higher prevalence of healthy dietary patterns in rural households and school areas. This contrast in international results demonstrates the clear differences in geographical profiles between the UK and North America and the resulting influence of geographical spaces determined by rurality on diet quality. While it is important that policy makers consider rurality and the potential effect that it may have on diet and therefore obesity and ill health, it is also important to remember that interventions may not be successfully transferred internationally due to cultural and geographical dissimilarities between countries.

While further research is required to support these findings in geographically diverse areas of the UK, our findings imply that interventions in the UK may have more impact in urban areas where poorer-quality dietary patterns are more prevalent and as such there may be more barriers to healthy diets. The increased association between rurality and nutrient-rich diets and the decreased association with snack foods (such as chocolate and crisps that are energy-dense and contribute few nutrients) suggest greater dietary health awareness in more rural areas. Our results also suggest that access to food in terms of geographical distance to food retail outlets (as observed by Smith et al. ${ }^{(17)}$ ) may not be a major influence on diet quality, as we would have expected to see consistent results between poorer access in rural areas and poorer diet quality if this was the case. It is plausible that other factors associated with families who live in rural areas (such as employment and income) are perhaps more relevant than access to food ${ }^{(41)}$. The results from our adjusted analyses suggest that previous arguments claiming neighbourhood characteristics play no significant role on diet quality after individual-level characteristics have been accounted for ${ }^{(42-44)}$ may not be valid. Although residual confounding may be an issue in our study, adjusting for individual factors such as maternal education and social class only slightly attenuated our effect sizes. Further research is needed to determine exactly what may be driving geographic variations in diet quality but our study indicates that differences do exist above the individual level.

The current study adds to the scant research examining associations between diet quality and rurality in the UK. The dietary variables used were well defined, identifying particular diet patterns known to have different nutrient profiles $^{(33,34)}$, and the inclusion of both PCA scores and dietary clusters allowed us to examine associations on two different measures. The data were from a large, generally representative sample of children from the UK. However, a number of limitations also exist with the study which must be acknowledged. First, it is only cross-sectional and as such it is not possible to determine causality. However, we consider reverse causality to be unlikely. Second, all dietary data were self-reported by the participants and as such may not have been completely accurate. While the use of nutritionists and interviews may have minimised errors in the data this is something that we should remain aware of. Third, small numbers in sparse areas restricted us from examining the effects of sparsity on dietary patterns. There is a possibility that the true associations between URI and diet quality may be underestimated due to the higher levels of drop out observed among participants living in increasingly urban areas. Finally, there is the possibility that underlying values of child rearing may exist in which people with healthier dietary patterns migrate to rural areas prior to pregnancy. However, such data are not available in the ALSPAC cohort, so we could not take this into account.

\section{Conclusion}

In conclusion, we have shown that geographical location in terms of rurality is associated with dietary patterns. The associations we report suggest that being resident in increasingly rural areas is linked to a higher prevalence of what may be considered healthier diets and a lesser tendency towards snack and processed foods; even after taking into account individual-level socio-economic confounding factors. Further work is needed to understand the mechanisms behind these differences, but the results presented here suggest that policy interventions to improve diet would be better targeted in urban than rural areas. While the cross-sectional nature of the research does not allow us to infer causality or direction of associations, our results are important for directing public health messages as they suggest that associations in diet quality truly exist at the spatial level and that rurality is itself independently linked to diet quality.

\section{Acknowledgements}

Acknowledgements: The authors are extremely grateful to all the families who took part in this study, the midwives for their help in recruiting them, and the whole ALSPAC team, which includes interviewers, computer and laboratory technicians, clerical workers, research scientists, volunteers, managers, receptionists and nurses. Financial support: The UK Medical Research Council and the Wellcome Trust (grant ref. 092731) and the University of 
Bristol provide core support for ALSPAC. The UK Medical Research Council, the Wellcome Trust and the University of Bristol had no role in the design, analysis or writing of this article. Conflict of interest: None. Autborship: Both authors contributed to the formulation of research questions, study design, interpretation of results and the final manuscript. Analysis was conducted by T.T.M. Ethics of buman subject participation: Ethical approval for the study was obtained from the ALSPAC Ethics and Law Committee and the Local Research Ethics Committees.

\section{References}

1. Sassi F (2010) Obesity and the Economics of Prevention: Fit Not Fat. Paris: OECD Publishing.

2. Parsons TJ, Power C, Logan S et al. (1999) Childhood predictors of adult obesity: a systematic review. Int J Obes Relat Metab Disord 23, Suppl. 8, S1-S107.

3. Mahmood T \& Arulkumaran S (2012) Obesity: A Ticking Time Bomb for Reproductive Health, 1st ed. London: Elsevier.

4. Jones N \& Lake I (2013) The combined impact of rural residence and socio-economic status on premature mortality. Health Place 24, 90-96.

5. Rayner M \& Scarborough P (2005) The burden of food related ill health in the UK. J Epidemiol Community Health 59, 1054-1057.

6. Hensrud DD (2004) Diet and obesity. Current Opin Gastroenterol 20, 119-124.

7. DiPietro L, Mossberg HO \& Stunkard AJ (1994) A 40-year history of overweight children in Stockholm: life-time overweight, morbidity, and mortality. Int $J$ Obes Relat Metab Disord 18, 585-590.

8. Mikkilä V, Räsänen L, Raitakari OT et al. (2005) Consistent dietary patterns identified from childhood to adulthood: the cardiovascular risk in Young Finns Study. Br J Nutr 93, 923-931.

9. Diez-Roux AV, Nieto FJ, Caulfield L et al. (1999) Neighbourhood differences in diet: the atherosclerosis risk in communities (ARIC) study. J Epidemiol Community Health 53, 55-63.

10. Van Hooijdonk C, Droomers M, Deerenberg IM et al. (2008) Higher mortality in urban neighbourhoods in The Netherlands: who is at risk? J Epidemiol Community Health 62 , 499-505.

11. House JS, Lepkowski JM, Williams DR et al. (2000) Excess mortality among urban residents: how much, for whom, and why? Am J Public Health 90, 1898-1904.

12. Cummins S \& Macintyre S (2006) Food environments and obesity - neighbourhood or nation? Int J Epidemiol 35, 100-104.

13. Black JL \& Macinko J (2008) Neighborhoods and obesity. Nutr Rev 66, 2-20.

14. Ellaway A \& Macintyre S (1996) Does where you live predict health related behaviours?: a case study in Glasgow. Health Bull (Edinb) 54, 443-446.

15. Shohaimi S, Welch A, Bingham S et al. (2004) Residential area deprivation predicts fruit and vegetable consumption independently of individual educational level and occupational social class: a cross sectional population study in the Norfolk cohort of the European Prospective Investigation into Cancer (EPIC-Norfolk). J Epidemiol Community Health 58, 686-691.

16. Macintyre S (2007) Deprivation amplification revisited; or, is it always true that poorer places have poorer access to resources for healthy diets and physical activity? Int J Behav Nutr Phys Act 4, 32.
17. Smith DM, Cummins S, Taylor M et al. (2010) Neighbourhood food environment and area deprivation: spatial accessibility to grocery stores selling fresh fruit and vegetables in urban and rural settings. Int J Epidemiol 39, 277-284.

18. Thornton LE, Crawford DA \& Ball K (2010) Neighbourhoodsocioeconomic variation in women's diet: the role of nutrition environments. Eur J Clin Nutr 64, 1423-1432.

19. Wrigley N \& Warm D (2003) Deprivation, diet, and foodretail access: findings from the Leeds 'food deserts' study. Environ Plann A 35, 151-188.

20. Rose D \& Richards R (2004) Food store access and household fruit and vegetable use among participants in the US Food Stamp Program. Public Health Nutr 7, 1081-1088.

21. McEachern MG \& Warnaby G (2006) Food shopping behaviour in Scotland: the influence of relative rurality. Int $J$ Consum Stud 30, 189-201.

22. Morland K, Diez Roux AV \& Wing S (2006) Supermarkets, other food stores, and obesity: the atherosclerosis risk in communities study. Am J Prev Med 30, 333-339.

23. Cummins S, Findlay A, Higgins C et al. (2008) Reducing inequalities in health and diet: findings from a study on the impact of a food retail development. Environ Plann A $\mathbf{4 0}$, 402-422.

24. Moore LV, Roux AVD, Nettleton JA et al. (2008) Associations of the local food environment with diet quality a comparison of assessments based on surveys and geographic information systems. Am J Epidemiol 167, 917-924.

25. Cummins S, Smith DM, Taylor M et al. (2009) Variations in fresh fruit and vegetable quality by store type, urban-rural setting and neighbourhood deprivation in Scotland. Public Health Nutr 12, 2044-2050.

26. Asirvatham J (2009) Examining diet quality and body mass index in rural areas using a Quantile Regression Framework. Rev Region Stud 39, 149-169.

27. Savoca MR, Arcury TA, Leng X et al. (2009) The diet quality of rural older adults in the South as measured by healthy eating index-2005 varies by ethnicity. J Am Diet Assoc 109, $2063-2067$.

28. Downs SM, Fraser SN, Storey KE et al. (2012) Geography influences dietary intake, physical activity and weight status of adolescents. Nutr Metab 2012, 816834.

29. Barker ME, McClean SI, Thompson KA et al. (1990) Dietary behaviours and sociocultural demographics in Northern Ireland. Br J Nutr 64, 319-329.

30. Morgan K, Armstrong GK, Huppert FA et al. (2000) Healthy ageing in urban and rural Britain: a comparison of exercise and diet. Age Ageing 29, 341-348.

31. Levin KA, Kirby J, Currie C et al. (2012) Trends in adolescent eating behaviour: a multilevel cross-sectional study of 11-15 year olds in Scotland, 2002-2010. J Public Health 34, 523-531.

32. Naska A, Fouskakis D, Oikonomou E et al. (2006) Dietary patterns and their socio-demographic determinants in 10 European countries: data from the DAFNE databank. Eur J Clin Nutr 60, 181-190.

33. Northstone K, Smith A, Newby P et al. (2013) Longitudinal comparisons of dietary patterns derived by cluster analysis in 7 to 13-year-old children. Br J Nutr 109, 2050-2058.

34. Smith AD, Emmett PM, Newby PK et al. (2013) Dietary patterns obtained through principal components analysis: the effect of input variable quantification. Br J Nutr 109, 1881-1891.

35. Boyd A, Golding J, Macleod J et al. (2013) Cohort profile: the 'Children of the 90s' - the index offspring of the Avon longitudinal study of parents and children. Int J Epidemiol 42, 111-127.

36. Avon Longitudinal Study of Parents and Children (2013) Data dictionary. http://www.bris.ac.uk/alspac/researchers/ data-access/data-dictionary/ (accessed January 2014). 
37. Cribb VL, Jones LR, Rogers IS et al. (2011) Is maternal education level associated with diet in 10-year-old children? Public Health Nutr 14, 2037-2048.

38. Office for National Statistics (2013) Output Areas (OA). http:// www.ons.gov.uk/ons/guide-method/geography/beginners-guide/census/output-area-oas-/index.html (accessed July 2013).

39. Office for National Statistics (2012) ONS Postcode Directory User Guide 2012 v4.1. https://geoportal.statistics.gov.uk/ geoportal/catalog/main/home.page (accessed September 2013)

40. Office of Population Censuses and Surveys (1990) Standard Occupational Classification. London: HMSO.
41. Dibsdall LA, Lambert N, Bobbin RF et al. (2003) Lowincome consumers' attitudes and behaviour towards access, availability and motivation to eat fruit and vegetables. Public Health Nutr 6, 159-168.

42. Cummins S \& Macintyre S (2002) A systematic study of an urban foodscape: the price and availability of food in Greater Glasgow. Urban Studies 39, 2115-2130.

43. Giskes K, Turrell G, van Lenthe FJ et al. (2006) A multilevel study of socio-economic inequalities in food choice behaviour and dietary intake among the Dutch population: the GLOBE study. Public Health Nutr 9, 75-83.

44. White M (2007) Food access and obesity. Obes Rev 8, 99-107. 